OPEN ACCESS

Edited by:

Yu Zhang,

Jinzhou Medical University, China

Reviewed by:

Eswari Dodagatta-Marni,

University of California, San Francisco,

United States

Talib Hassan Ali

University of ThiQar, Iraq

*Correspondence:

Wang $\mathrm{Li}$

liwang@sysucc.org.cn

tThese authors have contributed equally to this work

Specialty section:

This article was submitted to Molecular and Cellular Oncology,

a section of the journal

Frontiers in Oncology

Received: 02 December 2020 Accepted: 08 February 2021

Published: 23 March 2021

Citation:

Dai L, Huang Z and Li W (2021) Analysis of the PD-1 Ligands Among Gastrointestinal Cancer Patients:

Focus on Cancer Immunity.

Front. Oncol. 11:637015

doi: 10.3389/fonc. 2021.637015

\section{Analysis of the PD-1 Ligands Among Gastrointestinal Cancer Patients: Focus on Cancer Immunity}

\author{
Lin Dai ${ }^{1,2,3 \dagger}$, Zilin Huang ${ }^{4 \dagger}$ and Wang $\mathrm{Li}^{4 *}$ \\ ${ }^{1}$ Cancer Prevention Center, Sun Yat-sen University Cancer Center, Guangzhou, China, ${ }^{2}$ State Key Laboratory of Oncology in \\ South China, Guangzhou, China, ${ }^{3}$ Collaborative Innovation Center for Cancer Medicine, Guangzhou, China, ${ }^{4}$ Department of \\ Medical Imaging and Interventional Radiology, Sun Yat-sen University Cancer Center, Guangzhou, China
}

Many types of gastrointestinal cancer have shown promising outcomes after checkpoint blockade immunotherapy; however, it remains largely unclear about the expression profiles of programmed death 1 (PD-1) ligands (CD274 and PDCD1LG2) in the context of human pan-cancer. This work comprehensively analyzed the expression pattern of the PD-1 ligands and the clinical significance in the prognosis prediction among the seven types of gastrointestinal malignancies collected from The Cancer Genome Atlas (TCGA) and the Cancer Cell Line Encyclopedia (CCLE) database. Furthermore, the correlation of CD274/PDCD1LG2 with cancer immunity was also explored. The patients with liver hepatocellular carcinoma $(\mathrm{LIHC})$ receiving cytokine-induced killer $(\mathrm{CIK})$ cell immunotherapy at our cancer center were enrolled. CD274 and PDCD1LG2 displayed inconsistent gene expression levels among the diverse cancer cell lines. Typically, the abnormal expression level of CD274 and PDCD1LG2 was detected in both esophageal carcinoma (ESCA) and stomach adenocarcinoma (STAD), where PDCD1LG2 was related to the overall survival (OS) of the patients in ESCA $(p=0.015)$ and STAD $(p=$ 0.025). High-serum CD274 and PDCD1LG2 levels predicted a worse survival in the patients with $\mathrm{LIHC}$ receiving CIK therapy. More importantly, the expression level of CD274 and PDCD1LG2 was significantly correlated with the degree of Estimation of STromal and Immune cells in MAlignant Tumor tissues using the Expression data (ESTIMATE). In addition, we found that CD274 and PDCD1LG2 were correlated with gene markers in tumor-infiltrating immune cells. Furthermore, the expression of CD274 and PDCD1LG2 was correlated with tumor mutation burden (TMB), microsatellite instability (MSI), mismatch repair (MMR), and DNA methyltransferase (DNMT) of different types of cancers. The present work comprehensively analyzed a RNA sequencing of the PD-1 ligands across the seven distinct types of gastrointestinal cancers, which provided clues for further studies in cancer immunity and development.

Keywords: gastrointestinal cancer, CD274, PDCD1LG2, prognosis, cancer immunity 


\section{INTRODUCTION}

Gastrointestinal malignant tumor is a leading cause of death (1). The development of immunotherapy using immune checkpoint inhibitors has achieved breakthroughs in treating cancers. Programmed death 1 (PD-1) and its ligands, programmed death ligand 1 (PD-L1, or CD274) and PD-L2 (PDCD1LG2), are known as an immune checkpoint axis available to the cancer cells escaping from the immune destruction of $\mathrm{T}$ cells (2). In other words, PD-1 binds two ligands CD274 and PDCD1LG2 to inhibit the T-cell activation and invalidate immune surveillance. The results from experimental investigations and clinical studies showed that PD-1/PD-L1 blockers were demonstrated to be effective in gastrointestinal cancers $(3,4)$. However, a specific gene expression of CD274/PDCD1LG2 in gastrointestinal cancers at a pan-cancer level remains largely unknown.

A pan-cancer analysis is initially utilized in the field of cancer research to shed more light on the common features and heterogeneities of various human malignancies (5). A pancancer analysis, which is also referred to as an analysis of molecular abnormalities among several cancer types, is able to identify the common features and heterogeneities of some vital dysregulated biological processes in diverse cancer cell lineages. The project of a pan-cancer analysis, including Cancer Cell Line Encyclopedia (CCLE) and The Cancer Genome Atlas (TCGA), has been established based on the different human cancer cell lines and tissues at epigenomic, genomic, proteomic, and transcriptomic levels (6-8). Recently, a pan-cancer analysis is adopted to identify certain functional and pathway genes, which allows to specifically, comprehensively, and thoroughly understand human cancers. Considering as an example, the tumor hypoxia-associated multi-omic molecular characteristics were investigated, and some molecular alterations were suggested to be correlated with the sensitivity or resistance to antitumor agents. It contributed to a comprehensive understanding of tumor hypoxia at the molecular level and has certain implications for cancer treatment in clinical practice (9). The updated data regarding the frequency, etiology, and outcomes of the forkhead box protein M1 (FOXM1) upregulation in human cancers were defined among 33 cancer types derived from TCGA databases (10). Notably, the information obtained from these 33 TCGA-derived cancers had systemically revealed the long non-coding RNA- (lncRNA-) mediated dysregulation in cancers, which also provides the precious approach and resources to investigate the lncRNAs functions in cancers (11). It is beneficial to characterize the frequency of occurrence and variability of immune phenotypes within and among various cancer types,

\footnotetext{
Abbreviations: PD-1, programmed death 1; TCGA, The Cancer Genome Atlas; CCLE, Cancer Cell Line Encyclopedia; CIK, cytokine-induced killer; ESTIMATE, MAlignant Tumor tissues using the Expression data; TMB, tumor mutation burden; MSI, microsatellite instability; MMR, mismatch repair; DNMT, DNA methyltransferase; GTEx, Genotype-Tissue Expression; ROC, Receiver operating characteristic; KM, Kaplan-Meier; OS, overall survival; CHOL, cholangiocarcinoma; COAD, colon adenocarcinoma; ESCA, esophageal carcinoma; LIHC, liver hepatocellular carcinoma; PAAD, pancreatic adenocarcinoma; READ, rectum adenocarcinoma; STAD, stomach adenocarcinoma.
}

so as to understand the immune status for those untreated cancers. Typically, this method has been applied in more than 9,000 expression data of TCGA-derived cancer genes (12). Therefore, a pan-cancer analysis helps to illustrate the patterns that are beneficial for developing the combination treatments and individualized therapies.

In this regard, taking advantage of the large data sets from TCGA, this study aimed to examine the expression profiles of CD274/PDCD1LG2 and the prognostic significance in human gastrointestinal tumors. In addition, the association between CD274/PDCD1LG2 and the tumor infiltration level, tumor mutation burden (TMB), microsatellite instability (MSI), mismatch repair (MMR), and DNA methyltransferase (DNMT) were analyzed in the different tumor types. Our study helps to understand the vital roles of CD274/PDCD1LG2 in gastrointestinal tumor-immune interactions.

\section{MATERIALS AND METHODS \\ Patient Data Sets and Processing}

The Cancer Genome Atlas, a milestone of the cancer genomics project, characterizes thousands of primary cancer samples and matched the characterized samples with the adjacent noncarcinoma samples from seven types of cancers, including cholangiocarcinoma ( $\mathrm{CHOL}, N=45$ ), colon adenocarcinoma (COAD, $N=521$ ), esophageal carcinoma (ESCA, $N=173$ ), liver hepatocellular carcinoma (LIHC, $N=424$ ), pancreatic adenocarcinoma (PAAD, $N=182$ ), rectum adenocarcinoma (READ, $N=177$ ), and stomach adenocarcinoma (STAD, $N$ $=407)$. In this study, the TCGA level three RNA sequencing processed data and the corresponding clinical annotations were acquired by using the University of California Santa Cruz (UCSC) cancer genome browser (https://tcga.xenahubs. net, accessed April 2020). The gene expression data of healthy samples from the Genotype-Tissue Expression (GTEx) database (https://gtexportal.org/home/) was also downloaded for a comparison. The CCLE public project is established through a comprehensive characterization of tremendous human tumor models at both genetic and pharmacological levels. To examine the differential gene expression in cancers at a larger scale, the CCLE database containing the RNA-sequencing data sets for over 1,000 cell lines (https://portals.broadinstitute.org/ccle) was used in this study. Meanwhile, the approval from the Ethics Committee was exempted since only the open-access data were used. A total of 122 consecutive patients with LIHC receiving an adjuvant cytokine-induced killer (CIK) cell immunotherapy after a curative resection at our center were retrospectively enrolled, which were approved by the Ethics Committee of Sun Yatsen University Cancer Center. A detailed treatment workflow is described in our previous study (13).

\section{Gene Expression and Survival Analysis}

To compare the gene expression levels between cancer and adjacent non-carcinoma samples, the data regarding the gene expression profiles of CD274 and PDCD1LG2 were extracted from the seven cancer types in TCGA to form an expression matrix. Thereafter, the formed expression matrix and clinical 
information were matched with a patient ID. Receiver operating characteristic (ROC) curve was drawn to evaluate the ability of CD274 and PDCD1LG2 to distinguish the tumor sample from the normal sample. Moreover, the Kaplan-Meier (KM) analysis was conducted to compare the overall survival (OS) of the patients with TCGA cancer stratified based on the gene expression level of CD274 and PDCD1LG2 using a log-rank test.

\section{PD-1 Ligands and Tumor Immunity}

The Estimation of STromal and Immune cells in MAlignant Tumor tissues using the Expression data (ESTIMATE) is an approach that uses gene expression profiles to predict the tumor purity and the infiltrating stromal cells/immunocytes in tumor tissues (14). The ESTIMATE algorithm produces three scores based on the gene set enrichment analysis (GSEA) of a single sample, including (1) stromal score, which determines stromal cells in tumor tissues, (2) immune score, which stands for the immunocyte infiltration level in tumor tissues, and (3) estimate score, which infers the tumor purity. In this study, we used the ESTIMATE algorithm to estimate the immune and stromal scores in tumor tissues according to the corresponding transcriptional data. Later, we calculated the correlation of these scores with the expression of CD274 and PDCD1LG2.
Additionally, we examined the association of the expression level of CD274 and PDCD1LG2 with the gene markers in the selected tumor-infiltrating immunocytes with reference to the previous research studies (15-17). The estimated statistical significance and the Spearman's correlation coefficient were generated through a correlation analysis. Thereafter, we plotted an expression heatmap between a pair of genes in a specific type of cancer.

Tumor mutation burden measures the number of mutations in a specific cancer genome. Numerous articles have been conducted to explore the significance of using the TMB status as a biomarker to predict the patients with the highest response to checkpoint inhibitors (18). In this study, the somatic mutation data of all patients with TCGA were downloaded (https://tcga.xenahubs.net), their TMB scores were calculated, and the correlation between TMB and CD274/PDCD1LG2 was determined. MSI is characterized by the widespread length polymorphisms of microsatellite sequences resulting from DNA polymerase slippage. Recently, it was suggested that the patients with high MSI cancers can gain benefits from immunotherapy, and MSI was used as the genetic instability index for cancer detection (19). In this study, we calculated the MSI score for each patient, followed by a correlation analysis between MSI and CD274/PDCD1LG2. Notably, MMR, also referred to

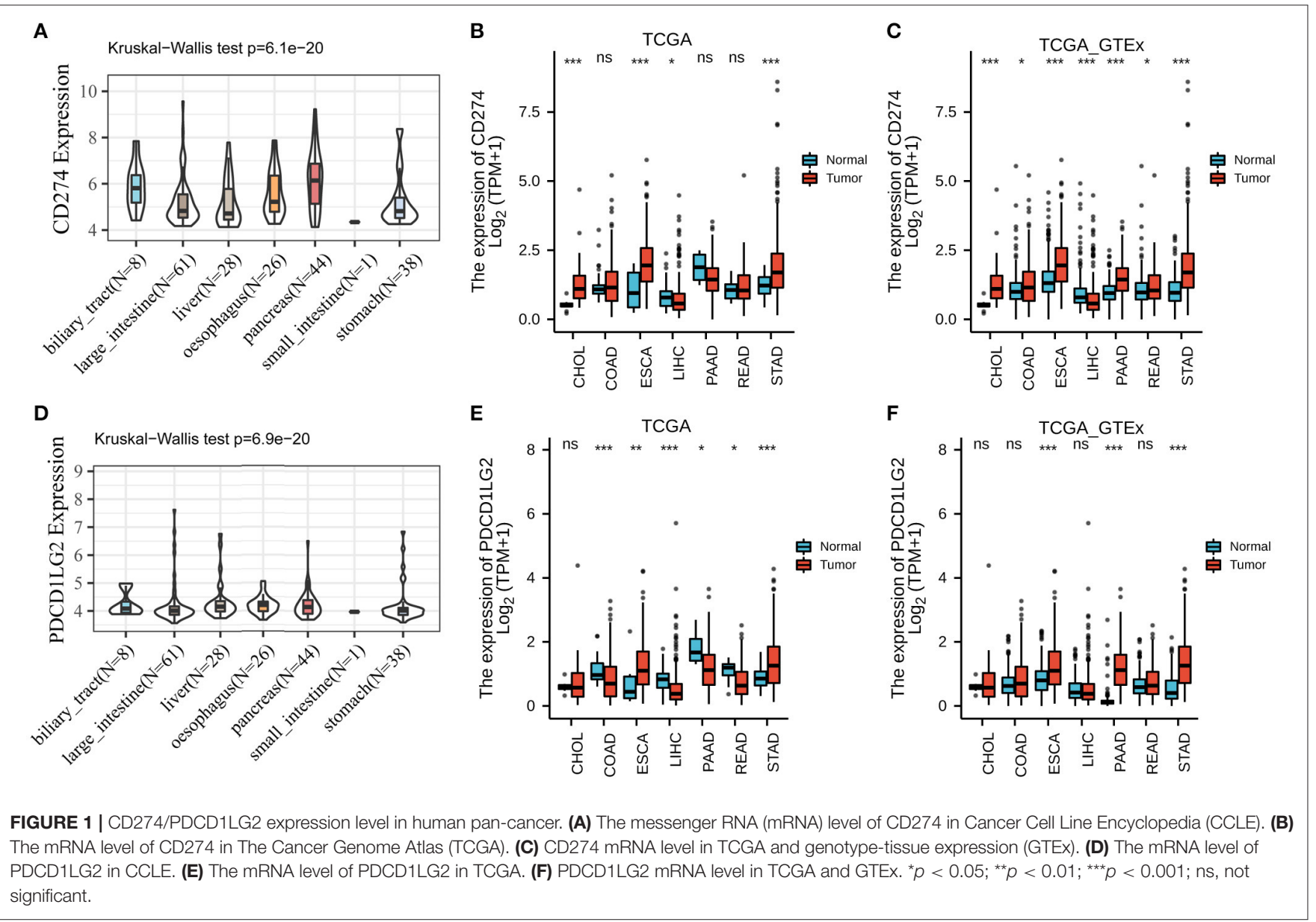



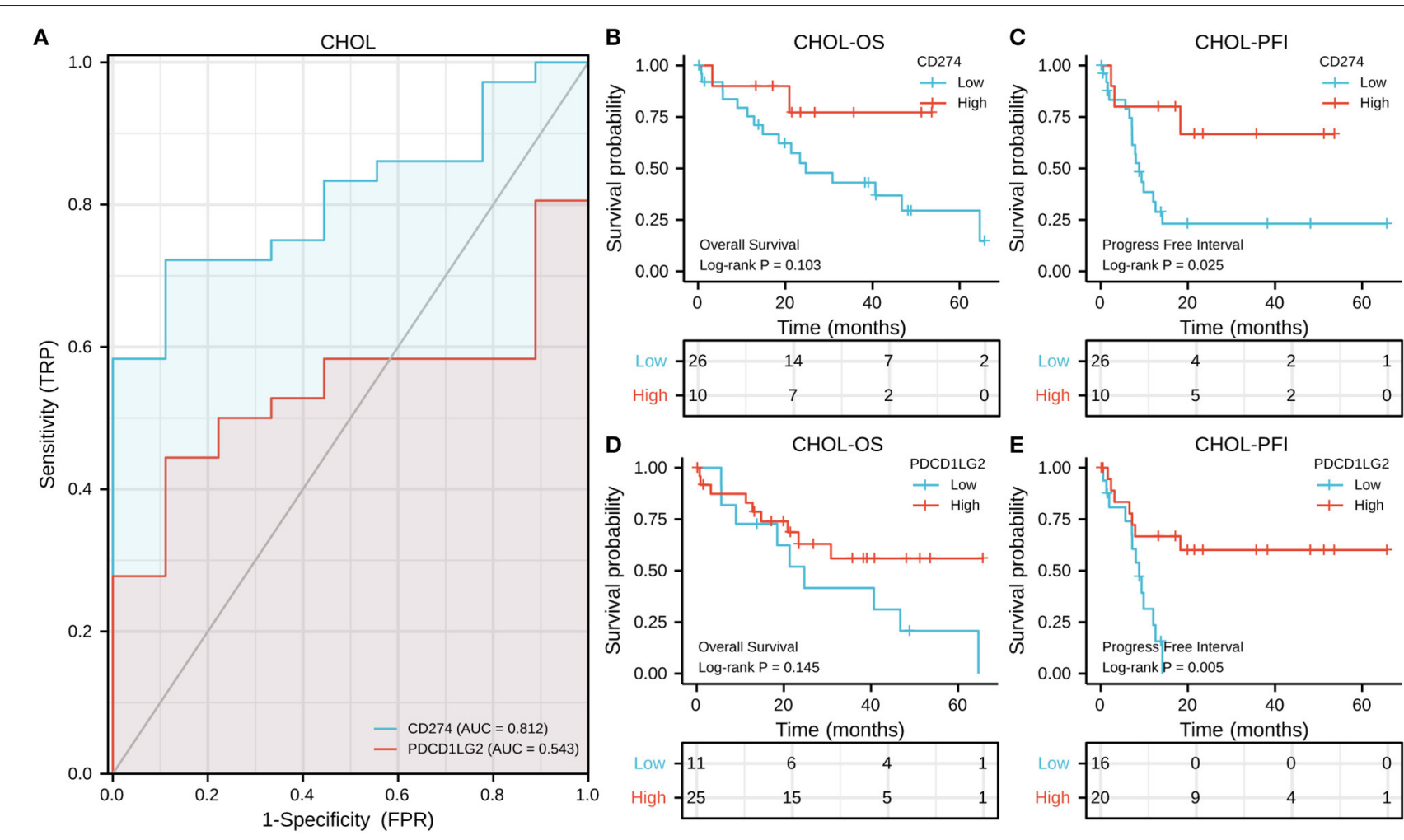

FIGURE 2 | CD274/PDCD1LG2 expression and its correlation with cholangiocarcinoma (CHOL) patient survival. (A) Receiver operating characteristic (ROC) curves CD274/PDCD1LG2 of distinguishing tumor from normal samples. (B,C) The Kaplan-Meier (KM) analysis shows the association between the CD274/PDCD1LG2 expression and overall survival (OS). (D,E) CD274/PDCD1LG2 expression and progression-free interval (PFI).

as the normal tissue DNA repair system, can correct errors in the process of DNA replication. However, due to the lack of MMR genes in tumor cells or the defects during the replication repair, the possibility of gene mutation has increased (19). Later, we performed a correlation analysis between MMR genes (including MLH1, MSH2, MSH6, PMS2, and EPCAM) and CD274/PDCD1LG2. DNMT participates in the process of tumorigenesis and development, whereas DNMT1, DNMT3A, and DNMT3B are the major key enzymes that catalyze DNMT (20). We also analyzed a correlation between DNMTs and CD274/PDCD1LG2.

\section{Statistical Analysis}

In the present work, the clinical survival types, including OS and progression-free interval (PFI), were selected for analysis. Generally, OS is deemed as the duration from the date of diagnosis to the date of death due to any cause, while PFI is defined as the disease progression or death from any cause.

The Wilcox log-rank test was adopted to determine the presence or absence of a markedly increased sum of the gene expression $\mathrm{z}$-scores in cancer tissues compared with the adjacent normal tissues. The area under the ROC curve was calculated to evaluate the ability of CD274 and PDCD1LG2 in distinguishing the tumor sample from the normal sample. Meanwhile, the Kruskal-Wallis test was employed to compare the difference in the expression of CD274 and PDCD1LG2 in cell lines. Survival was analyzed by the KM curves and a log-rank test. The Spearman test was utilized for a correlation analysis. The $\mathrm{R}$ language (version 3.6.0; $\mathrm{R}$ Foundation) was used for all analyses. A two-sided difference of $p<0.05$ indicated statistical significance.

\section{RESULTS}

\section{Pan-Cancer Expression Landscape of CD274 and PDCD1LG2}

According to the results of CCLE analysis, CD274 and PDCD1LG2 showed inconsistent gene expression levels among the diverse cancer cell lines $(p=6.1 \mathrm{e}-20$ and $6.9 \mathrm{e}-20$, Figures 1A,D), where pancreas cells had a relatively higher gene expression. Consistent with the different gene expression levels in CCLE, CD274, and PDCD1LG2 also displayed distinct expression in TCGA. For the seven TCGA-derived cancer types, we detected that ESCA, PAAD, and STAD showed a relatively higher gene expression. The expression landscapes of CD274 and PDCD1LG2 in TCGA cohorts are presented in Figures 1B,E, respectively. In addition, CD274 and PDCD1LG2 also exhibited a higher gene expression when more samples from GTEx were added Figures 1C,F. Figure 2A shows the AUC values of CD274 (0.812) and PDCD1LG2 (0.543) in the patients with CHOL, and the AUC results of the other six cancer types are presented in Supplementary Figures 1-6. 


\section{Screening of the Survival-Associated Cancers}

In the OS analysis, KM analysis showed that the subjects with high CD274 levels had short OS compared with those with low
CD274 levels in PAAD ( $p=0.012$, Supplementary Figure 4B) whereas those with an increased CD274 expression showed superior OS to those with a decreased CD274 expression in COAD ( $p=0.001$, Supplementary Figure 1B); the subjects

A
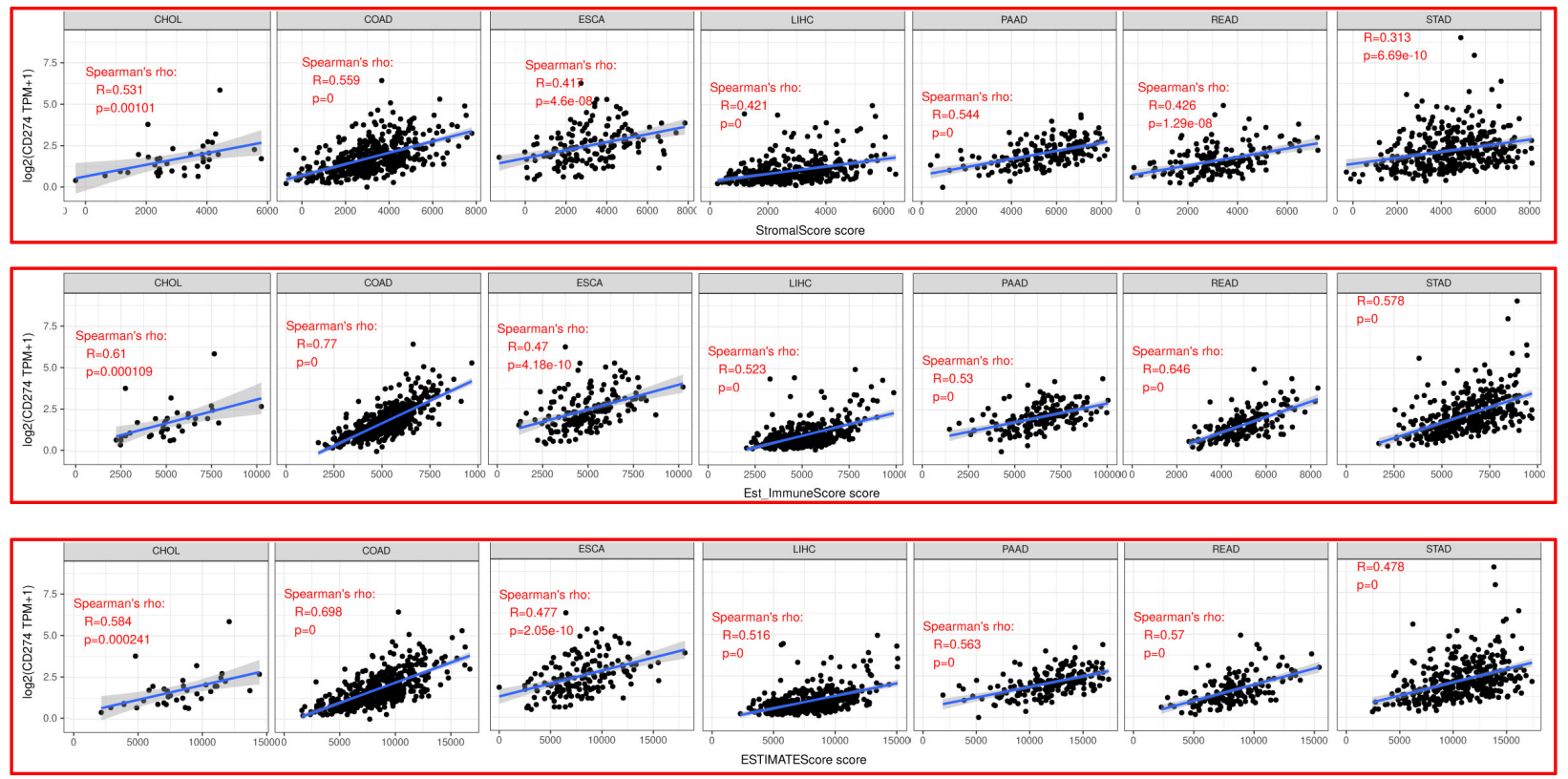

B
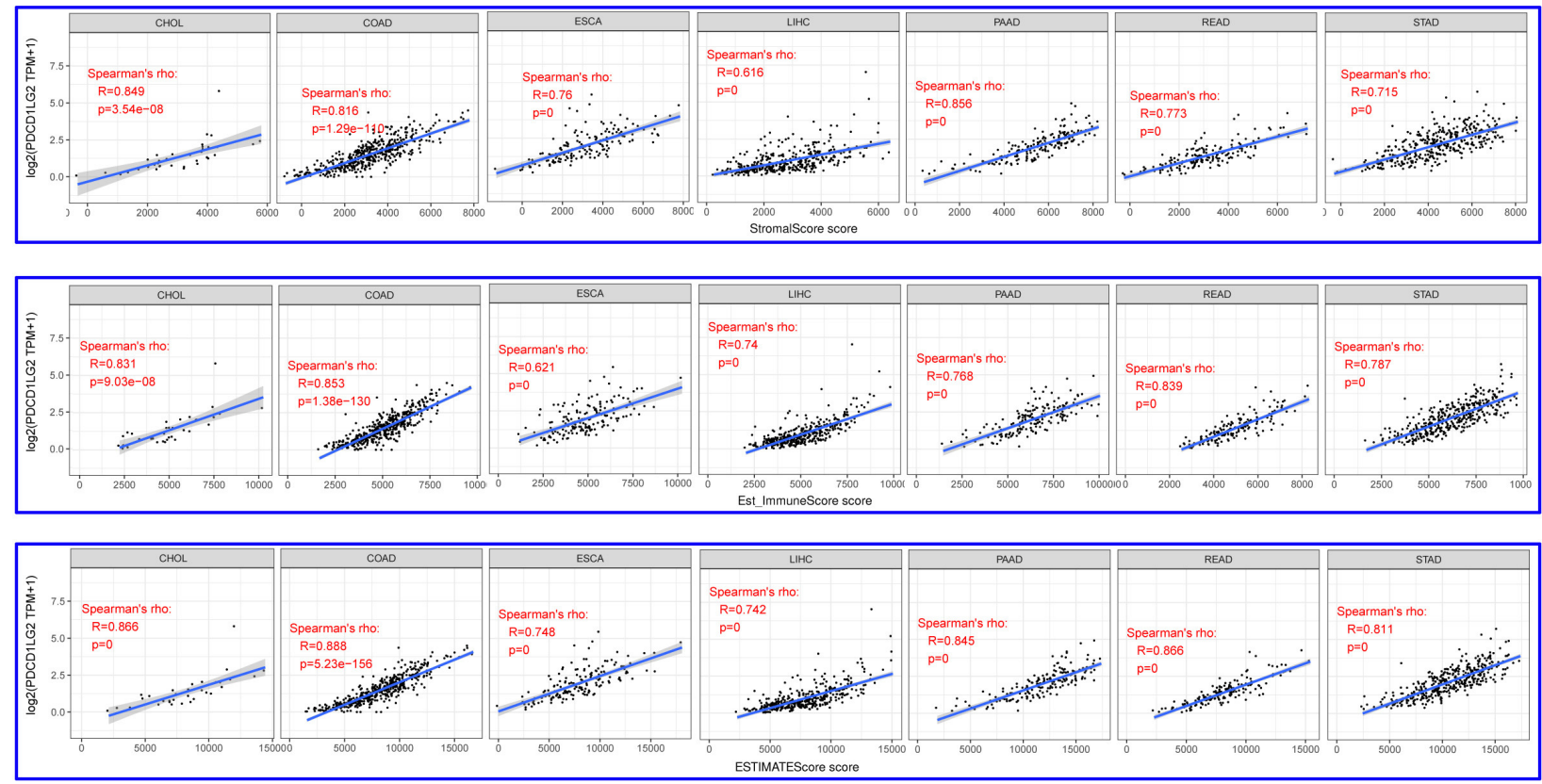

FIGURE 3 | CD274/PDCD1LG2 expression is correlated with cancer immunity in Estimation of STromal and Immune cells in MAlignant Tumor tissues using the Expression data (ESTIMATE). (A) ESTIMATE predicting CD274 expression is correlated with tumor immune infiltration level across seven cancer types. Top panel, stromal score; middle panel, immune score; and bottom panel, estimate score. (B) ESTIMATE predicting PDCD1LG2 expression is correlated with tumor immune infiltration level across seven cancer types. Top panel, stromal score; middle panel, immune score; and bottom panel, estimate score. 
with a high PDCD1LG2 expression had poorer OS than those with a low PDCD1LG2 expression in STAD $(p=0.025$, Supplementary Figure 6D) while the subjects with an increased
PDCD1LG2 expression showed superior OS to those with a decreased PDCD1LG2 expression in ESCA $(p=0.015$, Supplementary Figure 2D). In CHOL, no significant overall
A

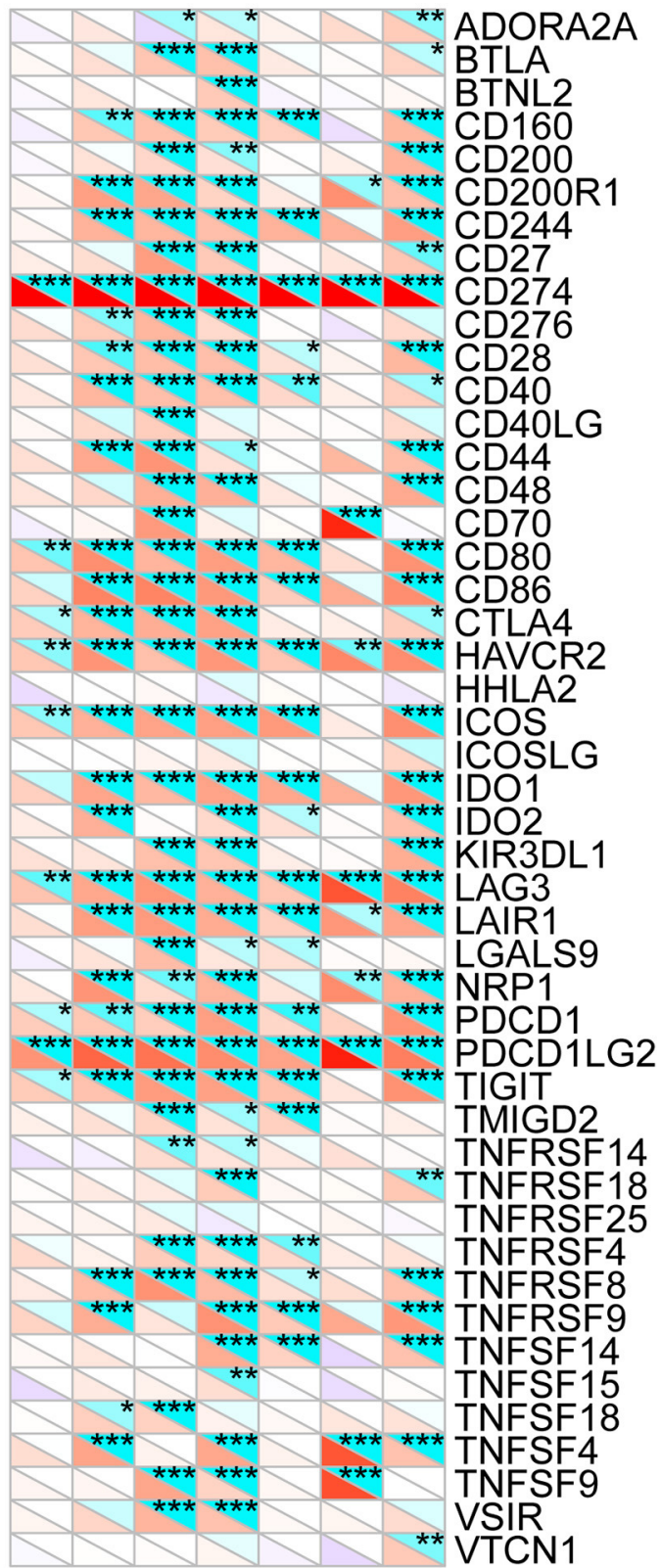

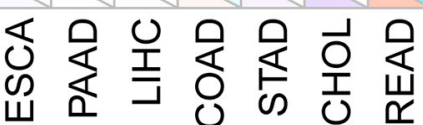

\begin{tabular}{llllllllll}
\multicolumn{2}{c}{ correlation } & \multicolumn{4}{c}{$-\log 10(p$ value) } \\
-1 & -0.5 & 0 & 0.5 & 1 & 0 & 0.75 & 1.5 & 2.2 & 3
\end{tabular}

B

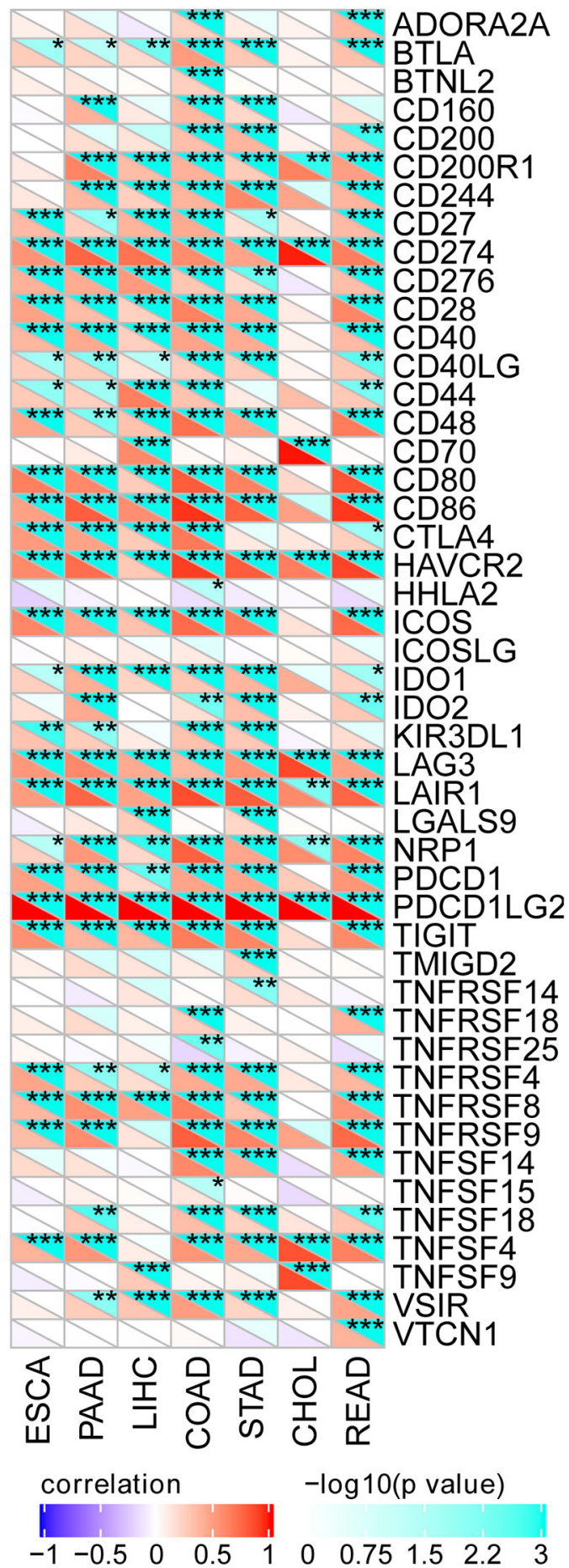

FIGURE 4 | CD274/PDCD1LG2 expression is correlated with immune markers. (A) Heatmap shows that the CD274 expression is correlated with the tumor immune marker level across seven cancers. For each pair, right top triangle is colored representing a $p$-value; left bottom is colored representing a correlation coefficient. (B) Heatmap shows that the PDCD1LG2 expression is correlated with the tumor immune marker level across seven cancers. ${ }^{*} p<0.05 ;{ }^{* *} p<0.01 ;{ }^{* \star *} p<0.001$. 
survival difference was seen between high and low gene expression patients (both $p$ values of CD274 and PDCD1LG2 were more than 0.05, Figures 2B,D).

In the PFI analysis, according to the results of KM analysis, the subjects with a high CD274 expression had poor PFI relative to those with a low $\mathrm{CD} 274$ expression in $\operatorname{PAAD}(p=$ 0.027, Supplementary Figure 4C); whereas the subjects having an increased CD274 expression showed superior PFI than those having a decreased CD274 expression in CHOL ( $p=$ 0.025, Figure 2C), as shown in Supplementary Figure 3; high PDCD1LG2 predicted superior prognosis for CHOL $(p=0.005$, Figure 2E) and LIHC ( $p=0.003$, Supplementary Figure 3E).

In our cancer center patients with LIHC, the median serum CD274 and PDCD1LG2 level were 5.1 and $14.7 \mu \mathrm{g} / \mu \mathrm{l}$, respectively. When patients were classified using the best statistical cutoff values $(\mathrm{CD} 274=11.22 \mu \mathrm{g} / \mu \mathrm{l}$ and PDCD1LG2
$=27.67 \mu \mathrm{g} / \mu \mathrm{l}$ ), survival analysis showed that the patients with LIHC with a high level of CD274 and PDCD1LG2 had worse disease-free survival after CIK immunotherapy (both $p<0.01$, Supplementary Figure 7).

\section{Expression of CD274/PDCD1LG2 Was Related to the Immune Infiltration Level}

The ESTIMATE method is developed to calculate the immune and stromal scores of cancer tissues. By adopting the ESTIMATE method, we calculated the immune, stromal, and estimate scores, respectively. Later, we evaluated the relationships between the immune/stromal scores and CD274/PDCD1LG2 expression. Figure 3 exhibits the results in these seven cancer types. Clearly, CD274/PDCD1LG2 expression was significantly correlated with the stromal, immune, and estimate scores (all values of $p<0.05$ ).
A

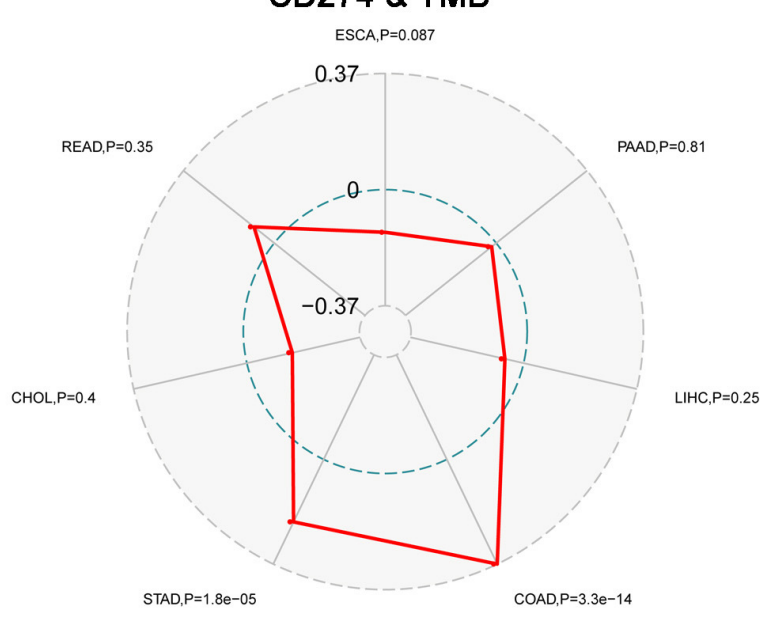

C

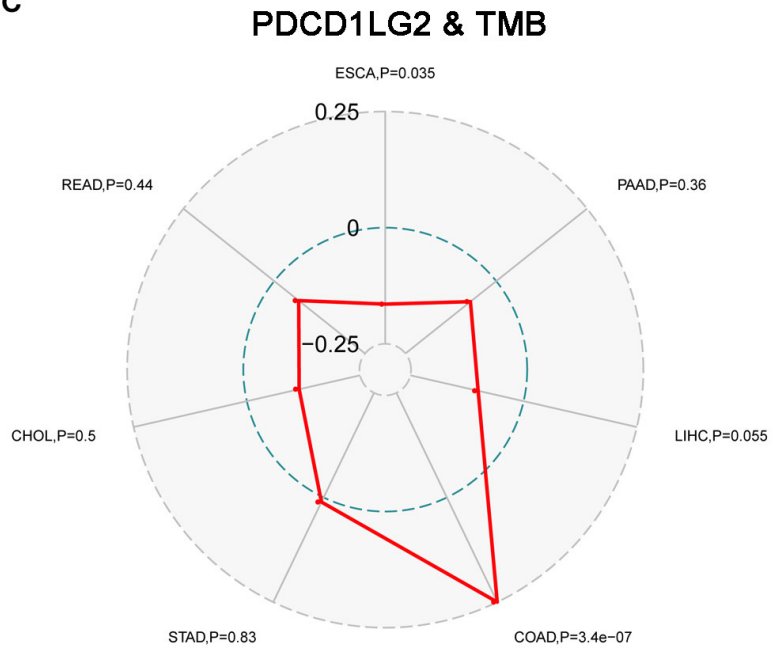

B

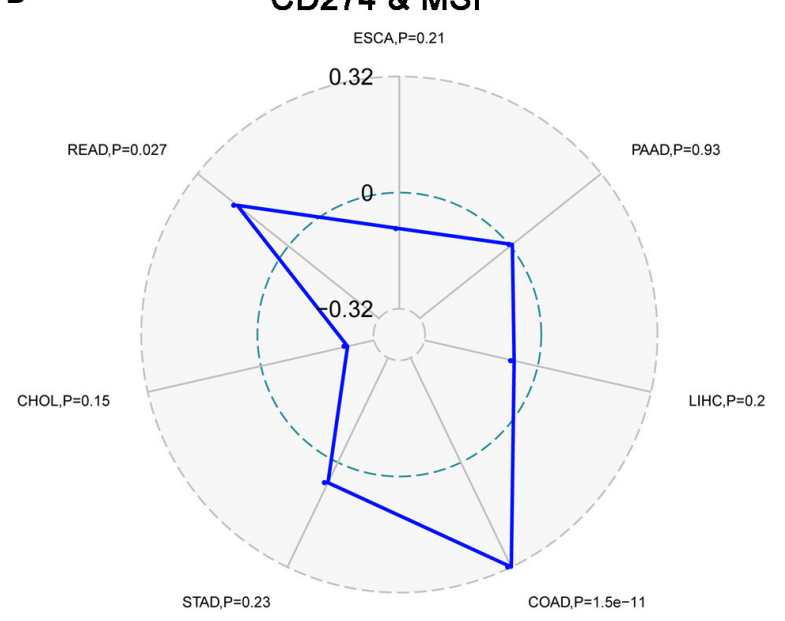

D

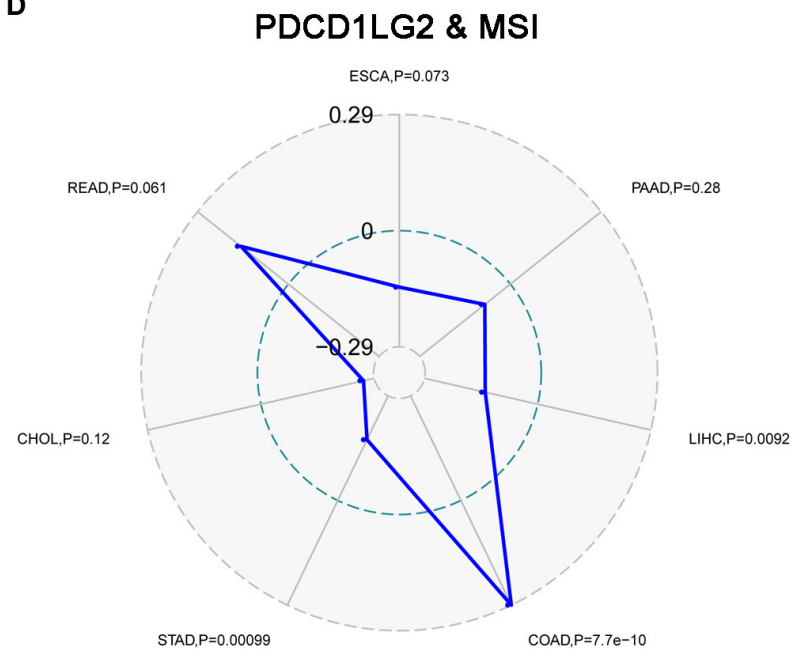

FIGURE 5 | CD274/PDCD1LG2 expression is correlated with tumor mutation burden (TMB) and microsatellite instability (MSI). (A) Radar chart displaying a correlation between CD274 and TMB across seven cancers. Red line representing the Spearman correlation coefficient. (B) Radar chart displaying a correlation between CD274 and MSI. Blue line representing the Spearman correlation coefficient. (C) PDCD1LG2 expression and TMB. (D) PDCD1LG2 and MSI. 


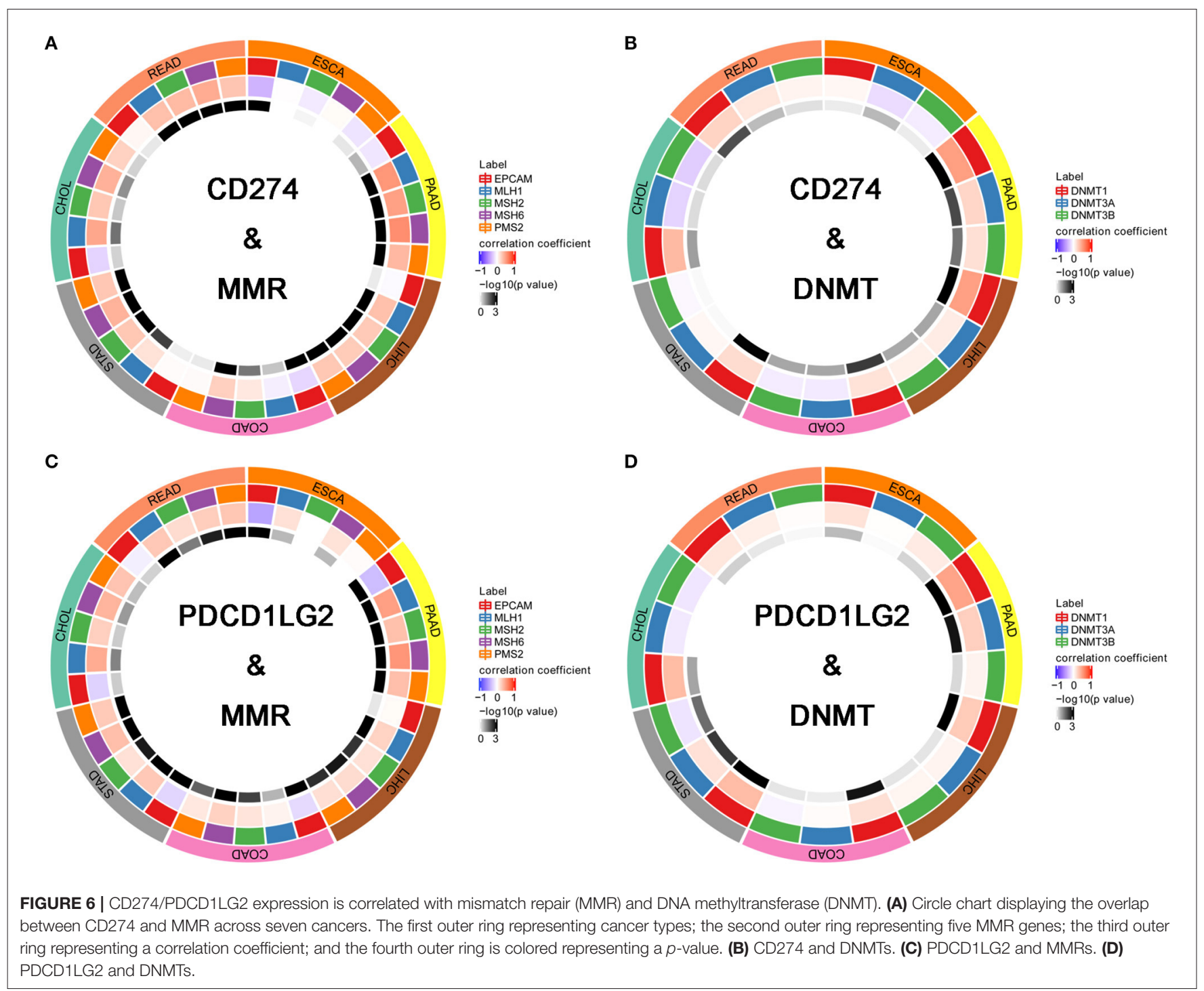

To better investigate the association of CD274/PDCD1LG2 expression with different immune infiltrating cells, we analyzed the relationship of CD274/PDCD1LG2 expression with the gene markers in diverse immunocytes, as shown in Figure 4. Our results suggested that CD274/PDCD1LG2 expression was significantly correlated with many immune markers in diverse immunocytes and distinct $\mathrm{T}$ cells.

\section{Correlation Analysis on TMB, MSI, MMR, and DNMT}

Moreover, we evaluated the association of TMB/MSI with CD274/PDCD1LG2 expression as shown in Figure 5. We discovered that the CD274 expression was correlated with the TMB in STAD $(p=1.8 \mathrm{e}-5)$, and $\operatorname{COAD}(p=3.3 \mathrm{e}-14)$; while PDCD1LG2 expression was correlated with the TMB in ESCA ( $p$ $=0.035)$, and COAD $(p=3.4 \mathrm{e}-7)$, as presented in Figures 5A,C. Moreover, CD274 expression was found to be related to the MSI in $\operatorname{COAD}(p=1.5 \mathrm{e}-11)$, and $\operatorname{READ}(p=0.027)$; whereas
PDCD1LG2 expression was associated with the MSI in COAD ( $p$ $=7.7 \mathrm{e}-10)$, LIHC $(p=0.009)$, and STAD $(p=0.001)$, as displayed in Figures 5B,D.

Further, we performed a correlation analysis between MMR genes (MLH1, MSH2, MSH6, PMS2, and EPCAM) and CD274/PDCD1LG2 as shown in Figures 6A,C. In all seven cancer types, CD274/PDCD1LG2 was correlated with at least one MMR gene. Besides, we also conducted a correlation analysis between DNMT (DNMT1, DNMT2, DNMT3A, and DNMT3B) and CD274/PDCD1LG2. As a result, in all seven cancer types, CD274/PDCD1LG2 was correlated with at least one DNMT gene (Figures 6B,D). Detailed correlation results are summarized in Table 1.

\section{DISCUSSION}

The present work illustrated a comprehensive workflow for a pan-cancer analysis and thoroughly investigated the 
TABLE 1 | Correlation analysis of CD274/PDCD1LG2 expression with mismatch repair (MMR) and DNA methyltransferase (DNMT).

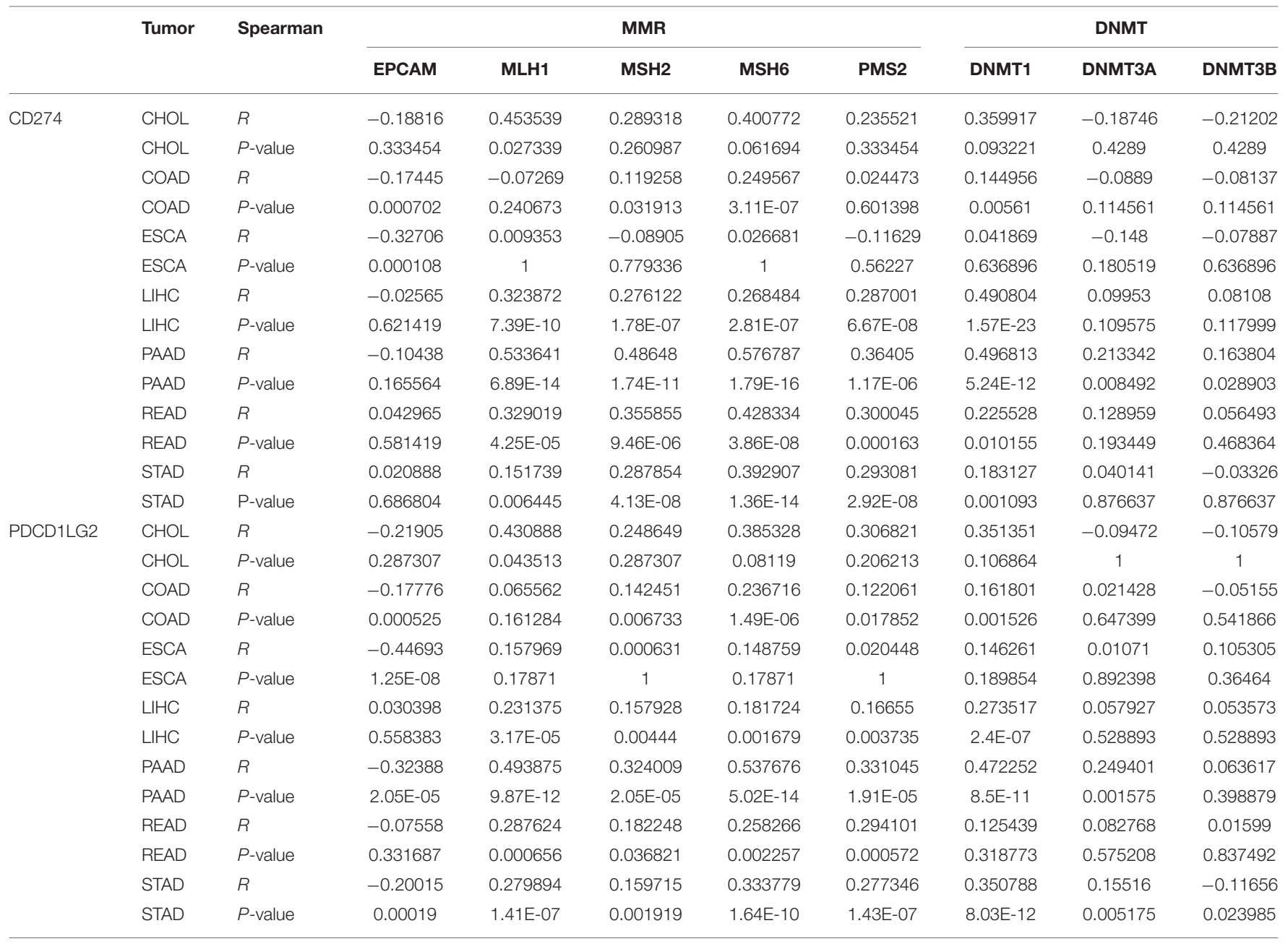

role of CD274/PDCD1LG2 in gastrointestinal cancers. The CD274/PDCD1LG2 expression among the different cancer cell lines was reported. It was found that most cancer types showed a CD274/PDCD1LG2 alteration frequency, and the abnormal expression served as a prognostic factor in some cancer types. Serum CD274/PDCD1LG2 levels could predict disease-free survival after the CIK cell therapy in patients with LIHC. More importantly, the CD274/PDCD1LG2 expression was associated with the cancer immunity. Furthermore, CD274/PDCD1LG2 was identified to be correlated with TMB, MSI, MMR, and DNMT.

It is significant to identify the abnormal gene expression among the different types of cancers. Also, it is of great significance to identify the tumor-specific targets or tumorrelated features in individualized treatment, thus enhancing the possibility of curing among the patients with cancer (21). A pancancer analysis of CD274/PDCD1LG2 is valuable for identifying a differential expression and its role in many cancer types $(22,23)$. Using CCLE and TCGA databases, a large number of diverse types of cancers are obtained, which contributes to discovering the abnormal CD274/PDCD1LG2 expression among the different types of cancers. Besides, a thorough pan-cancer cellular analysis of gene expression can be performed through CCLE, which sheds light on the future cellular experiments. On the other hand, TCGA genomic and survival analyses may provide guidance for clinical practice and future studies.

In recent years, immunotherapy has achieved prominent efficacy in the treatment of tumors. Notably, the present work also demonstrated that the expression of CD274/PDCD1LG2 was related to cancer immunity. According to this study, the CD274/PDCD1LG2 level was related to the immune infiltration levels in cancers. ESTIMATE has been reported as a metric for the evaluation of the prognosis for the patients with cancer (24). Numerous recent studies have employed the ESTIMATE method to assess various tumors, and it has been successfully applied to the genome data. For instance, ESTIMATE is used to predict the outcomes among the patients with glioblastoma and cutaneous melanoma $(25,26)$. Using a TCGA cohort, the ESTIMATE method was utilized to generate the immune and stromal scores. As a result, CD274/PDCD1LG2 was correlated with the ESTIMATE scores. Furthermore, CD274/PDCD1LG2 was also detected to be 
correlated with the gene markers in infiltrating cells as observed from Figure 4.

Gene mutation is a major cause of cancer formation (27). Typically, mutations in some specific genes may predict the patient prognosis and treatment response $(28,29)$. An adaptive immune system can identify and detect cancers through the nonself neoantigens associated with somatic mutations. Therefore, TMB affects the possibility of generation of an immunogenic peptide, thus impacting the patient response to immune checkpoint inhibitors $(30,31)$. Consequently, it is of great significance to comprehensively investigate the association of the CD274/PDCD1LG2 expression with TMB levels among the patients with cancer, using the TCGA-derived high-quality matched data. Moreover, TMB and MSI also indicate the production of new antibodies. Noteworthily, a number of cases with high MSI (MSI-H) show increased TMB levels (32). Further, as discovered by Bonneville et al. (33), the MSI-H cervical squamous cell carcinoma and adrenocortical carcinoma showed abnormally high mutation frequencies. On the other hand, MSI is a vital index to predict the tumor genesis and development (19). The National Comprehensive Cancer Network (NCCN) guidelines have recommended an MSI testing for all READ subtypes, and the READ mortality can be reduced by the early detection of MSI (34). Some studies have shown that the MMR defect (dMMR) in cancer cells produces antigens that can be easily recognized by $\mathrm{T}$ cells, in this way, the $\mathrm{PD}-1$ inhibitors are highly effective on the MSI-H solid tumors (35). In this regard, FDA has approved the use of Keytruda for the treatment of MSI-H/dMMR solid tumors (36). Therefore, TMB, MSI, and MMR can serve as a predicting factor for the efficacy of immunotherapy at present. We found in this study that the CD274/PDCD1LG2 expression was correlated with TMB and MSI in some cancer types. Moreover, the gene DNA methylation can regulate gene expression, while such a change can be utilized by tumor cells to destroy the immunogenicity and the immune recognition mechanisms, thus obtaining the immune escape phenotypes. Notably, the application of a methylase inhibitor in combination with an immunocheckpoint inhibitor has achieved initial advantages (20). An analysis between CD274/PDCD1LG2 and a methylase inhibitor is expected to inject a new vitality into the cancer treatment. Taken together, our findings provide clues for the association between CD274/PDCD1LG2 and cancer immunity, which require further experiment investigations to clarify.

\section{REFERENCES}

1. Bray F, Ferlay J, Soerjomataram I, Siegel RL, Torre LA, Jemal A. Global cancer statistics 2018: GLOBOCAN estimates of incidence and mortality worldwide for 36 cancers in 185 countries. CA: A cancer J Clin. (2018) 68:394-424. doi: 10.3322/caac.21492

2. Syn NL, Teng MWL, Mok TSK, Soo RA. De-novo and acquired resistance to immune checkpoint targeting. Lancet Oncol. (2017) 18:e73141. doi: 10.1016/S1470-2045(17)30607-1

3. Charoentong P, Finotello F, Angelova M, Mayer C, Efremova $M$, Rieder D, et al. Pan-cancer immunogenomic analyses reveal genotype-immunophenotype relationships and predictors of
Collectively, our comprehensive pan-cancer analysis has characterized CD274/PDCD1LG2 in different cancer cell lines and tissues of seven gastrointestinal cancer types. Our new integrative omics-based workflow might provide novel insights into the patients with gastrointestinal cancer regarding immunotherapy.

\section{DATA AVAILABILITY STATEMENT}

The datasets presented in this study can be found in online repositories. The names of the repository/repositories and accession number(s) can be found in the article/Supplementary Material.

\section{AUTHOR CONTRIBUTIONS}

WL conceived of and designed the study, supervised the study, and reviewed the manuscript. $\mathrm{LD}$ and $\mathrm{ZH}$ performed the literature search, collected and analyzed the data, generated the figures and tables, and wrote the manuscript. All authors contributed to the article and approved the submitted version.

\section{SUPPLEMENTARY MATERIAL}

The Supplementary Material for this article can be found online at: https://www.frontiersin.org/articles/10.3389/fonc. 2021.637015/full\#supplementary-material

Supplementary Figure 1 | CD274/PDCD1LG2 expression and its correlation with colon adenocarcinoma (COAD) patient survival.

Supplementary Figure 2 | CD274/PDCD1LG2 expression and its correlation with esophageal carcinoma (ESCA) patient survival.

Supplementary Figure 3 | CD274/PDCD1LG2 expression and its correlation with liver hepatocellular carcinoma $(\mathrm{LIHC})$ patient survival.

Supplementary Figure 4 | CD274/PDCD1LG2 expression and its correlation with pancreatic adenocarcinoma (PAAD) patient survival.

Supplementary Figure 5 | CD274/PDCD1LG2 expression and its correlation with rectum adenocarcinoma (READ) patient survival.

Supplementary Figure 6 | CD274/PDCD1LG2 expression and its correlation with stomach adenocarcinoma (STAD) patient survival.

Supplementary Figure 7 | Serum CD274/PDCD1LG2 level and its correlation with disease free survival in LIHC CIK patients. (A) Serum CD274 (PDL1) distribution and cutoff of PDL1 level. (B) Lower serum PDL1 level was associated with longer disease free survival. (C,D) PDCD1LG2 (PDL2). response to checkpoint blockade. Cell Rep. (2017) 18:24862. doi: 10.1016/j.celrep.2016.12.019

4. Liu JN, Kong XS, Huang T, Wang R, Li W, Chen QF. Clinical implications of aberrant PD-1 and CTLA4 expression for cancer immunity and prognosis: a pan-cancer study. Front Immunol. (2020) 11:2048. doi: 10.3389/fimmu.2020.02048

5. Cancer Genome Atlas Research N, Weinstein JN, Collisson EA, Mills GB, Shaw KR, Ozenberger BA, et al. The cancer genome atlas pan-cancer analysis project. Nat Gene. (2013) 45:1113-20. doi: 10.1038/ng.2764

6. Li W, Chen QF, Huang T, Shen L, Huang ZL, Wu P. Profiles of m(6)A RNA methylation regulators for the prognosis of hepatocellular carcinoma. Oncol Lett. (2020) 19:3296-306. doi: 10.3892/ol.2020.11435 
7. Huang ZL, Li W, Chen QF, Wu PH, Shen LJ. Eight key long non-coding RNAs predict hepatitis virus positive hepatocellular carcinoma as prognostic targets. World J Gastrointest Oncol. (2019) 11:983-97. doi: 10.4251/wjgo.v11.i11.983

8. Cancer Cell Line Encyclopedia C, Genomics of Drug Sensitivity in Cancer C. Pharmacogenomic agreement between two cancer cell line data sets. Nature. (2015) 528:84-7. doi: 10.1038/nature15736

9. Ye Y, Hu Q, Chen H, Liang K, Yuan Y, Xiang Y, et al. Characterization of hypoxia-associated molecular features to aid hypoxia-targeted therapy. Nat Metab. (2019) 1:431-44. doi: 10.1038/s42255-019-0045-8

10. Barger CJ, Branick C, Chee L, Karpf AR. Pan-cancer analyses reveal genomic features of FOXM1 overexpression in cancer. Cancers. (2019) 11:251. doi: 10.3390/cancers11020251

11. Li Y, Li L, Wang Z, Pan T, Sahni N, Jin X, et al. LncMAP: Pan-cancer atlas of long noncoding RNA-mediated transcriptional network perturbations. Nucleic Acids Res. (2018) 46:1113-23. doi: 10.1093/nar/gkx1311

12. Danaher P, Warren S, Lu R, Samayoa J, Sullivan A, Pekker I, et al. Pan-cancer adaptive immune resistance as defined by the Tumor Inflammation Signature (TIS): results from The Cancer Genome Atlas (TCGA). J Immunother Cancer. (2018) 6:63. doi: 10.1186/s40425-018-0367-1

13. Chang B, Huang T, Wei H, Shen L, Zhu D, He W, et al. The correlation and prognostic value of serum levels of soluble programmed death protein 1 (sPD-1) and soluble programmed death-ligand 1 (sPD-L1) in patients with hepatocellular carcinoma. Cancer Immunol Immunother. (2019) 68:35363. doi: 10.1007/s00262-018-2271-4

14. Yoshihara K, Shahmoradgoli M, Martinez E, Vegesna R, Kim H, Torres-Garcia W, et al. Inferring tumour purity and stromal and immune cell admixture from expression data. Nat Commun. (2013) 4:2612. doi: $10.1038 /$ ncomms 3612

15. Siemers NO, Holloway JL, Chang H, Chasalow SD, Ross-MacDonald $\mathrm{PB}$, Voliva $\mathrm{CF}$, et al. Genome-wide association analysis identifies genetic correlates of immune infiltrates in solid tumors. PLoS ONE. (2017) 12:e0179726. doi: 10.1371/journal.pone.0179726

16. Danaher P, Warren S, Dennis L, D'Amico L, White A, Disis ML, et al. Gene expression markers of tumor infiltrating leukocytes. J Immunother Cancer. (2017) 5:18. doi: 10.1186/s40425-017-0215-8

17. Pan JH, Zhou H, Cooper L, Huang JL, Zhu SB, Zhao XX, et al. LAYN is a prognostic biomarker and correlated with immune infiltrates in gastric and colon cancers. Front Immunol. (2019) 10:6. doi: 10.3389/fimmu.2019.00006

18. Krieger T, Pearson I, Bell J, Doherty J, Robbins P. Targeted literature review on use of tumor mutational burden status and programmed cell death ligand 1 expression to predict outcomes of checkpoint inhibitor treatment. Diagnostic Pathol. (2020) 15:6. doi: 10.1186/s13000-020-0927-9

19. Li K, Luo H, Huang L, Luo H, Zhu X. Microsatellite instability: a review of what the oncologist should know. Cancer Cell Int. (2020) 20:16. doi: 10.1186/s12935-019-1091-8

20. Dan H, Zhang S, Zhou Y, Guan Q. DNA methyltransferase inhibitors: catalysts for antitumour immune responses. OncoTargets Ther. (2019) 12:1090316. doi: 10.2147/OTT.S217767

21. Andre F, Mardis E, Salm M, Soria JC, Siu LL, Swanton C. Prioritizing targets for precision cancer medicine. Ann Oncol. (2014) 25:2295303. doi: 10.1093/annonc/mdu478

22. Cao Z, Zhang S. An integrative and comparative study of pan-cancer transcriptomes reveals distinct cancer common and specific signatures. Sci Rep. (2016) 6:33398. doi: 10.1038/srep33398

23. Cava C, Bertoli G, Colaprico A, Olsen C, Bontempi G, Castiglioni I. Integration of multiple networks and pathways identifies cancer driver genes in pan-cancer analysis. BMC Genom. (2018) 19:25. doi: 10.1186/s12864-017-4423-x

24. Liu W, Ye H, Liu YF, Xu CQ, Zhong YX, Tian T, et al. Transcriptome-derived stromal and immune scores infer clinical outcomes of patients with cancer. Oncol Lett. (2018) 15:4351-7. doi: 10.3892/ol.2018.7855

25. Jia D, Li S, Li D, Xue H, Yang D, Liu Y. Mining TCGA database for genes of prognostic value in glioblastoma microenvironment. Aging. (2018) 10:592605. doi: 10.18632/aging.101415

26. Yang $S$, Liu $T$, Nan $H$, Wang $Y$, Chen $H$, Zhang $X$, et al. Comprehensive analysis of prognostic immune-related genes in the tumor microenvironment of cutaneous melanoma. J Cell Physiol. (2020) 235:1025-35. doi: 10.1002/jcp.29018

27. Martincorena I, Campbell PJ. Somatic mutation in cancer and normal cells. Science. (2015) 349:1483-9. doi: 10.1126/science.aab4082

28. Sanz-Garcia E, Argiles G, Elez E, Tabernero J. BRAF mutant colorectal cancer: prognosis, treatment, and new perspectives. Ann Oncol. (2017) 28:264857. doi: 10.1093/annonc/mdx401

29. Allegra CJ, Rumble RB, Hamilton SR, Mangu PB, Roach N, Hantel $A$, et al. Extended RAS gene mutation testing in metastatic colorectal carcinoma to predict response to anti-epidermal growth factor receptor monoclonal antibody therapy: American society of clinical oncology provisional clinical opinion update 2015. J Clin Oncol. (2016) 34:17985. doi: 10.1200/JCO.2015.63.9674

30. Wu HX, Wang ZX, Zhao Q, Chen DL, He MM, Yang LP, et al. Tumor mutational and indel burden: a systematic pan-cancer evaluation as prognostic biomarkers. Ann Translat Med. (2019) 7:640. doi: $10.21037 / \mathrm{atm} .2019 .10 .116$

31. Havel JJ, Chowell D, Chan TA. The evolving landscape of biomarkers for checkpoint inhibitor immunotherapy. Nat Rev Cancer. (2019) 19:13350. doi: 10.1038/s41568-019-0116-x

32. Chalmers ZR, Connelly CF, Fabrizio D, Gay L, Ali SM, Ennis R, et al. Analysis of 100,000 human cancer genomes reveals the landscape of tumor mutational burden. Genome Med. (2017) 9:34. doi: 10.1186/s13073-017-0424-2

33. Bonneville R, Krook MA, Kautto EA, Miya J, Wing MR, Chen HZ, et al. Landscape of microsatellite instability across 39 cancer types. JCO Precis Oncol. (2017) PO.17.00073. doi: 10.1200/PO.17.00073

34. Benson AB III, Venook AP, Cederquist L, Chan E, Chen YJ, Cooper HS, et al. Colon cancer, version 1.2017, NCCN clinical practice guidelines in oncology. J Natl Comprehen Cancer Netw: JNCCN. (2017) 15:370-98. doi: 10.6004/jnccn.2017.0036

35. Diaz LA Jr, Le DT. PD-1 blockade in tumors with mismatch-repair deficiency. N Engl J Med. (2015) 373:1979. doi: 10.1056/NEJMc1510353

36. Yu Y. Molecular classification and precision therapy of cancer: immune checkpoint inhibitors. Front Med. (2018) 12:22935. doi: $10.1007 / \mathrm{s} 11684-017-0581-0$

Conflict of Interest: The authors declare that the research was conducted in the absence of any commercial or financial relationships that could be construed as a potential conflict of interest.

Copyright (c) 2021 Dai, Huang and Li. This is an open-access article distributed under the terms of the Creative Commons Attribution License (CC BY). The use, distribution or reproduction in other forums is permitted, provided the original author(s) and the copyright owner(s) are credited and that the original publication in this journal is cited, in accordance with accepted academic practice. No use, distribution or reproduction is permitted which does not comply with these terms. 